\title{
Embedded Spectrum Sensor Network Architecture and Transmission Medium Test Based on TCP/IP
}

\author{
http://dx.doi.org/10.3991/ijoe.v12i05.5734 \\ Ya Zhang ${ }^{1}$ and Fang Han $^{2}$ \\ ${ }^{1}$ Changzhou College of Information Technology, Changzhou, China \\ ${ }^{2}$ Huainan Normal University, Huainan, China
}

\begin{abstract}
Electromagnetic spectrum monitoring that fully covers spectrum resources guarantees the orderly use of key technologies. However, existing monitoring equipment and methods cannot satisfy the increasing demand. A feasible solution for this problem is a wireless sensor network that is developed using a wireless self-organized network technology for a new type of distributed information system. For the wireless spectrum-monitoring network used by software, this study analyzes the operating system and hardware platform and then tests the physical transport layer under different transmission rates. Factors that may affect transmission performance are also investigated. A two-layer network model is eventually used based on the network to adapt to the requirements and the minimum transmission rate. The first layer uses cable, wideband code-division multiple access (CDMA), and time-division synchronous CDMA; the second layer network uses Wi-Fi/cable. This two-layer network model combines two heterogeneous networks, thereby enabling perception among nodes and gateway nodes. The management center can smooth the data transmission process. To perform several tasks simultaneously, we design our own two layers at the application layer protocol, namely, transmission protocol and data. The twostage ad-hoc network process, the working principle, and the implementation method of each network application layer module are analyzed. The new spectrum sensor network structure exhibits low power consumption, low cost, as well as distributed and self-organization characteristics. It can guarantee the safety of data transmission with a good transmission rate.
\end{abstract}

Index Terms-TCP/IP, network module function realization, wireless sensor network design

\section{INTRODUCTION}

A wireless sensor network (WSN) is a large network system that can realize highly efficient and reliable communication among nodes. An embedded operating system (EOS) refers to a complete set of operating system software with a considerably extensive application scope. The transmission control protocol (TCP)/Internet protocol (IP) is the most widely used Internet basic agreement. This agreement is divided into four layers; the subsequent layer interface is provided by the agreement to complete the function of this layer.

Scientists and scholars have achieved considerable research results to address this problem. In contrast to the general perspective of a traditional network, WSN is designed according to different objectives, and thus, has a strong application background. In the sensor network operation process, the nodes in a network perform a series of procedures, such as collaborative perception of information, monitoring, collection, etc.; after data are processed, they are returned to the service side [1]. The characteristics of WSN are appropriate for military reconnaissance, particularly as a means of accessing information. The task area is sufficient for distribution network nodes, the positions of which are not fixed and are highly random. Therefore, WSN is suitable for modern battlefield environments. The demand of the military for the electromagnetic spectrum is increasing, and gathering enemy information in such complex environments is particularly important [2]. Mainstream EOSs available in the market, such as Linux, Windows, and IOS, typically include system kernel file, hardware, software, interface, and network communication protocol [3]. An embedded system provides key kernel free customization, strong stability, high real-time capability, etc. [4]. The main characteristic of TCP/IP is not only its capability to work on specific network architecture but also its strong openness and capability to connect different heterogeneous networks. For unified network address allocation, the standardization of a high-level protocol can provide a wide range of reliable user services to ensure that each TCP/IP device has a unique address in the same network [5].

This paper mainly reports on a model for spectrummonitoring network based on an embedded platform, which applies the independent research and development of a laboratory-constructed network protocol in an actual network. The second chapter of this article presents general technology theory of the embedded TCP/IP spectrum sensor. The third chapter discusses technological innovations. In the fourth chapter, an innovative technology is used to build an embedded spectrum sensor. Data are also analyzed in this chapter. Finally, the study is concluded in the fifth chapter.

\section{STATE OF THE ART}

\section{A. System Hardware Platform}

For the control center of this system, particularly the Acorn RISC machine (ARM) chip and the fieldprogrammable gate array (FPGA) chip, the software system adopts the Linux operating system. The FPGA chip is mainly responsible for spectrum data acquisition and processing, whereas the ARM chip is responsible for handling the EOS and application program. For the specific model, namely, the AT91SAM9G45 processor, the chip 
kernel is ARM926EJ-s (Figure 1). This processor supports various mainstream flash memory interfaces and is equipped with a special double external bus interface. The capacity of the distributed memory is $64 \mathrm{k}$. These interfaces support the development of good communication with external devices [6].

AT91SAM9G45 has an operating frequency of a 400 $\mathrm{MHZ}$ processor and a multiple data transfer rate of over 100 Mbps peripherals; hence, it exhibits extremely high performance and network bandwidth or local memory bandwidth that are sufficient to provide good user experience. The QY-9 g45ek AT91SAM9G45 development board from Arcelormittal Electronic Science and Technology perfectly supports multiple operating systems, such as Linux, wince system, etc. Such systems are widely used in industrial control, medical electronics, energy conservation, environmental protection, intelligent transportation, mining terminals, power systems, communication systems, the textile industry, automotive electronics, industrial touch screen control systems, robot visuals, media processing, wireless applications, digital home appliances, automotive equipment, communication equipment, and network terminal conditions.

AT91SAM9G45 supports the program and data storage of the latest generation of DDR2 and NAND flash memory interface. An internal $133 \mathrm{MHz}$ multi-layer bus architecture with 37 direct memory access (DMA) channels, a dual external bus interface, and distributed storage, including a $64 \mathrm{kB}$ static random-access memory that can be configured as a tightly coupled memory. The bus architecture can maintain the ? of the processor as well as the high speed and high bandwidth requirements of the peripherals. Input/output (I/O) supports a working voltage of $1.8 \mathrm{~V}$ or $3.3 \mathrm{~V}$ for the memory interface, peripheral $\mathrm{I} / \mathrm{O}$, and the separate configuration. Through this feature, the user will not require any external level shifter. This feature also supports 0.8 spacing encapsulation, and thus, can achieve low-cost printed circuit board manufacturing. The AT91SAM9G45 power management controller contains a clock door (clock gating) and backup battery parts. This controller can be activated and can minimize power consumption during standby mode. AT91SAM9G45 supports SD card start burning and can easily perform customer system update and batch production test. An internal highspeed USB2.0 interface chip can be realized in TianMo technology SBC6845 board with $12 \mathrm{MB}$ read and write operations. This chip provides a good network controller and DMA communication structure, which can realize high-speed network communication. The network speed measured in the SBC6845 board in terms of actual communication velocity is over $90 \mathrm{MB}$. The application of this convenient and reliable interface can play a prominent role in directing industrial communication. The AT91SAM9G45 serial port controller has an RS485 communication mode function, which can realize accurate application of RS485. Accurate switching can be implemented as system communication overhead and inconvenience are reduced. Double bus architecture is one of the features of the chip; it does not affect operation under minimum system condition. DMA function, system stability, and communication system efficiency lead to a good performance.

The aforementioned architecture is mainly used in industrial control terminals, intelligent instruments, data collection and analysis, health careproducts, remote me-

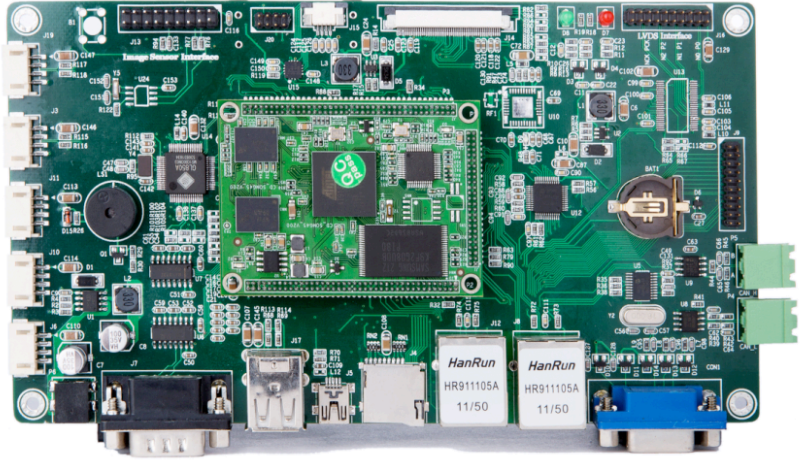

Figure 1. Schematic of hardware development board

ter-reading systems, concentrators, and network terminals, among others.

\section{B. Linux EOS}

This study uses the Linux EOS as a WSN node of the operating system. The Linux operating system provides good multitasking support and efficient control efficiency. Moreover, the difficulty of development is moderate. Linux is based on a Unix operating system, and it inherits the network characteristics of this system as its core. The Linux operating system uses the main platform of the $x 86$ computer for its $32 / 64$ operation platform, which provides excellent support [7].

The current Linux system has a high market share and excellent user experience rating. Compared with other operating systems, Linux has several advantages. First, Linux is a free, open-source software; thus, considerable development costs are saved. Second, Linux has a strong purpose. Developers customize systems and Linux can be used according to different backgrounds to modify the kernel, thereby making this operating system efficient and require minimal memory. The application of the Linux platform is also efficient.

In the past, embedded development used assembly language, and thus, readability was extremely poor. The complicated development process required using such assembly language to write a driver for all interfaces and hardware. The Linux system was then introduced. Linux installs approximately 2 million bytes of memory system and can accomplish the aforementioned task.

Linux memory management: Memory management of all the operating systems is one of the most important aspects of the memory management mechanism; it basically determines the efficiency and stability of a system. Linux has a good memory management mechanism, i.e., it accomplishes time-sharing multitasking through the paging mechanism.

Linux file system: This study uses the Linux file system. However, the mobile terminal is the mainstream of the file system, characteristic is to read and write speed. The Linux operating system supports many file systems, such as the virtual file system, to achieve data interaction.

The Linux process and thread management aim to achieve many-to-one threads and processes using methods similar to Solaris, which causes the system to treat threads and processes in the same manner. Linux can replicate via the command function process, i.e., the process of fatherand-son sharing resources. The Linux process and thread can be assumed to be similar because of this mechanism. 
PAPER

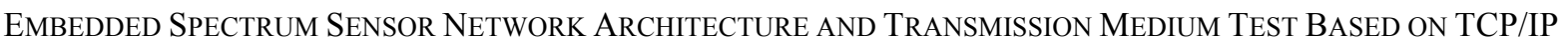

The advantages of the Linux EOS are its efficient customization and excellent network performance. In contrast to the traditional operating system, the Linux EOS can support TCP/IP better. The Linux platform has a sufficient driver for support. Linux uses the identification process affiliate program priority level, which makes the system reliable and reduces its development cost.

\section{METHODOLOGY}

TCP/IP is divided into four layers, namely, the interface, network, transport, and application layers. This protocol has a good failure recovery mechanism, does not interrupt existing services to join a network, and exhibits efficient error rate data-processing capability, platform independence, and low cost.

TCP/IP has been originally designed to provide national security. When a part of a network is damaged, its other parts can still work normally. This protocol operates fast and requires simple implementation. Hence, TCP/IP has gradually become the most widely used network protocol.

TCP/IP works in the network layer transport protocol. IP has matured after years of development. The current mainstream supports IP network equipment. IP provides a connectionless data service and is responsible for communication among computers. Its function can be extended in the near future. IP is not a connection-oriented transfer protocol; thus, it cannot guarantee the integrity of the arriving data. TCP/IP secures data transmission completely through a layer of agreement to ensure that IP alone provides affordable transport services.

Among the four layers of TCP/IP, TCP works at the transport layer. Different hosts in a network typically establish a reliable connection at the application layer. This layer will be used in the network transmission of data to a lower level. TCP divides the data into message segment lengths to fulfill the requirements (length by the media access control layer in the network of maximum transmission unit restrictions). After break up, the TCP data are segmented into the IP layer, which transfers the data through the network to the destination host. TCP assigns each byte number in advance, with a serial number to ensure that the packets sent are in order and to prevent packet loss. The destination after the packet is successfully received will confirm back characters (ACK). If the sending host rules in the packet round-trip delay have not yet received confirmation within characters, then lost packets will be retransmitted. TCP uses a checksum function to check whether data have errors. Sending and receiving checks are calculated.

The development of a TCP link is divided into three steps. First, the machines at the sending end send a request packet, in addition to the SYN flag bit with no other datum, before connection is established to the receiver. Second, the receiver machine receives the SYN packet, and the confirmation contains only the SYN + ACK packet. Third, the first sending machine confirms the receiver in the second step after the packet returns and sends the ACK packet to the receiver machine as the final confirmation. Machines perform data communication thrice, and a communication link is considered the reliable basic setup.

The User Datagram Protocol (UDP) does not require packet data transmission. In strict accordance with the order that arrives, the packet sequences of inspection and sorting are completed after data transmission is completed by the application layer. However, UDP alone has an unreliable information service.

The workflow of UDP can be described as follows. The local machine receives a UDP packet from the network. The solution package obtains the port number of the UDP packet, and the machine performs a task queuing and message port number matching process. If the task queue and port number are matched, then UDP receives the end of the local task queue that is waiting to be processed. If no criterion exists, the source address of the Internet is discarded based on UDP to reply to a message.

TCP and UDP are the transport layers in TCP/IP. TCP data transmission is required to establish a network link. UDP performs transmission preparation, and the overhead setup link is considerable. Although TCP is a reliable transport protocol, IP does not guarantee the reliability of data transmission. TCP alone has excellent error detection and retransmission mechanisms that effectively solve transmission errors. TCP is a streaming protocol. UDP does not need to establish a network link, its resource overhead is small, and it transmits data rapidly. However, UDP has no fixed communication link, and thus, it does not guarantee the integrity and reliability of data.

$\mathrm{TCP} / \mathrm{IP}$, as the basis of current Internet use, generally has positive and extensive applications. TCP/IP in various designs is presented to solve the problem of Internet working on heterogeneous networks. This protocol is designed as a point-to-point protocol on both sides of the IP address of IP packets. Data transmission occurs in the same physical network devices within a network. However, IP alone does not guarantee transmission reliability. TCP/IP can control the subnet of a machine. It does not depend on a specific hardware or operating system and sensor characteristics. This study designs a network for reliable data transmission and speed, which has certain requirements. The entire system can combine heterogeneous networks with one-to-one or one-to-many control and data transmission based on the aforementioned reasons and the characteristics of sensor network applications. This study uses TCP/IP stack.

TCP/IP uses four transports as a network interface, namely, cable, Wi-Fi, wideband code-division multiple access (WCDMA), and time-division synchronous codedivision multiple access (TD-SCDMA), as shown in Figure 2.

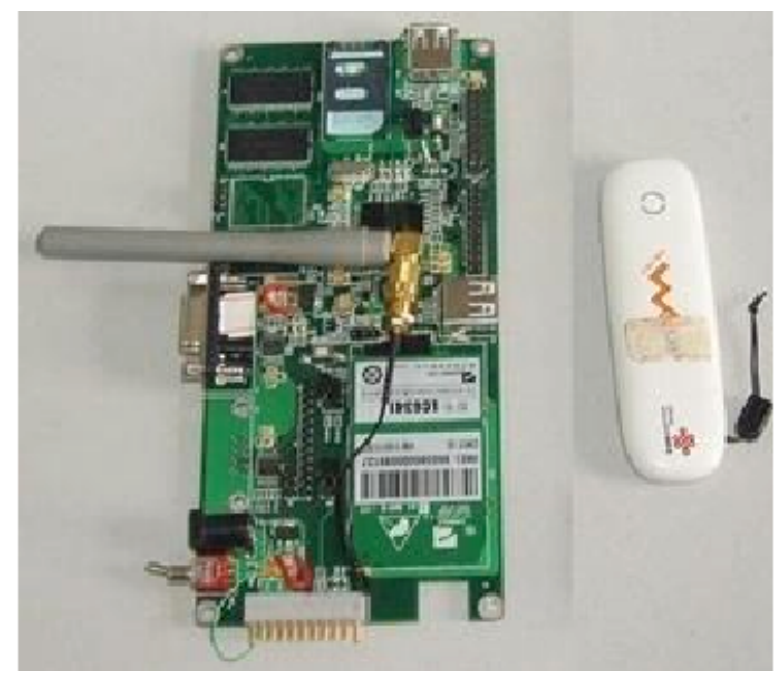

Figure 2. TD-SCDMA and WCDMA equipment 
To enable transmission rate and quality to satisfy the demand and to achieve the goal of a fast network within a short period, the perception nodes of network equipment mainly use Wi-Fi/cable (primarily for indoor test) transmission because far-distance perception nodes typically exist in the management center and the position of perception nodes may change. Table 1 lists the Wi-Fi used in our test, the speed of the transmission module test, and the data used in the Wi-Fi module for performance analysis. Figures 3 and 4 show the data histogram.

From the data, we determine that the Wi-Fi transfer rate may affect signal strength, equipment, and moving objects, such as Wi-Fi antenna orientation [8]. For data communication between two devices, a close distance leads to high signal strength and good transmission reliability [9]. The network connection is normal during the data testing process. Smooth transmission during the entire test prevents network congestion or link break. The transmission rate can reach $12 \mathrm{Mbps}$, which can completely satisfy the demand.

\section{RESUlt ANALYSIS AND DisCUSSION}

Figure 5 shows the flowchart of the implementation in the application layer protocol in network architecture.

This system adopts two-stage, three-layer networks. The two stages belong to a heterogeneous network, the network management center, and the gateway, which directly uses a $3 \mathrm{~g} /$ cable network, a sensor network, and a Wi-Fi network.

The application layer protocol designed in this study can be divided into two layers, namely, business protocol and transport protocol. The transport protocol specifies the format of the application layer in Baotou. Gateway nodes forward cluster-head node command. The business protocol specifies the specific contents of the application layer packet data entity part, the data entity store command parameters, or the contents of the returned data; these data are based on the agreement according to a certain format stored in a data entity within the business; when the receiving end receives the packet, it can also regulate the business protocol data entity [10].

TABLE I.

WI-FI TEST RESULTS

\begin{tabular}{|l|l|l|l|l|l|l|l|l|}
\hline & \multicolumn{2}{|l}{$\mathbf{d B i}$} & \multicolumn{1}{l|}{$\mathbf{1 2} \mathbf{d B i}$} \\
\hline $\begin{array}{l}\text { Distance } \\
\boldsymbol{m}\end{array}$ & $\begin{array}{l}\text { Level } \\
\boldsymbol{d B m}\end{array}$ & $\begin{array}{l}\text { Rate } \\
\text { Mbps }\end{array}$ & $\begin{array}{l}\text { Maximum rate } \\
\text { Mbps }\end{array}$ & $\begin{array}{l}\text { Minimum rate } \\
\text { Mbps }\end{array}$ & $\begin{array}{l}\text { Level } \\
\boldsymbol{d B m}\end{array}$ & $\begin{array}{l}\text { Rate } \\
\text { Mbps }\end{array}$ & $\begin{array}{l}\text { Maximum rate } \\
\text { Mbps }\end{array}$ & $\begin{array}{l}\text { Minimum rate } \\
\text { Mbps }\end{array}$ \\
\hline 60 & -79 & 21.3 & 30 & 26 & -76.6 & 19.4 & 31.3 & 2.8 \\
\hline 450 & -69.4 & 18.32 & 24.1 & 11.4 & -67 & 22.28 & 27.8 & 17 \\
\hline 240 & -72 & 1.81 & 4.0 & 0.649 & -78 & 11.25 & 15.0 & 8.1 \\
\hline
\end{tabular}

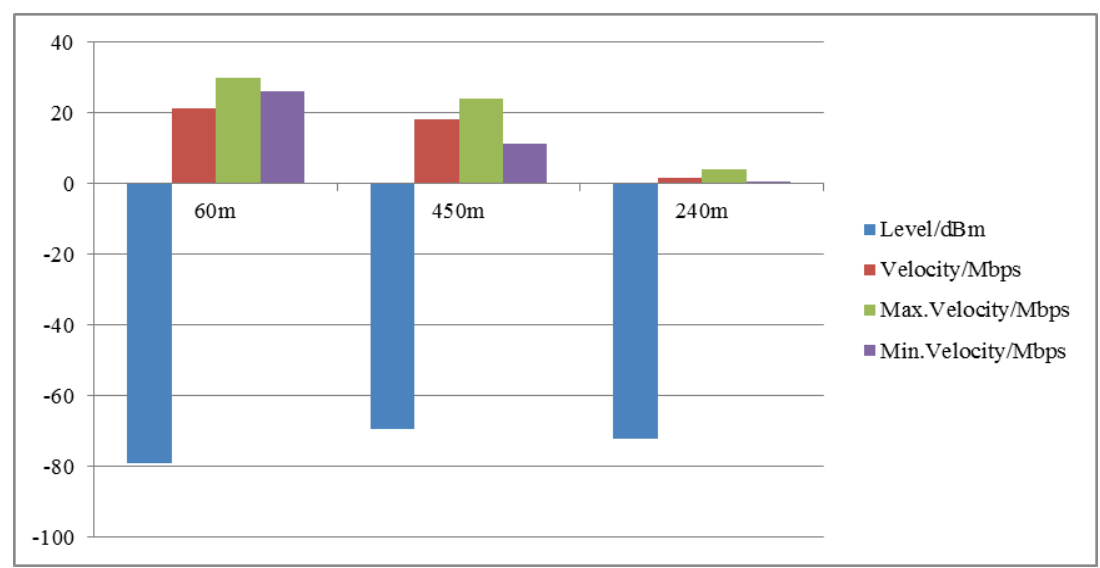

Figure 3. Wi-Fi test results

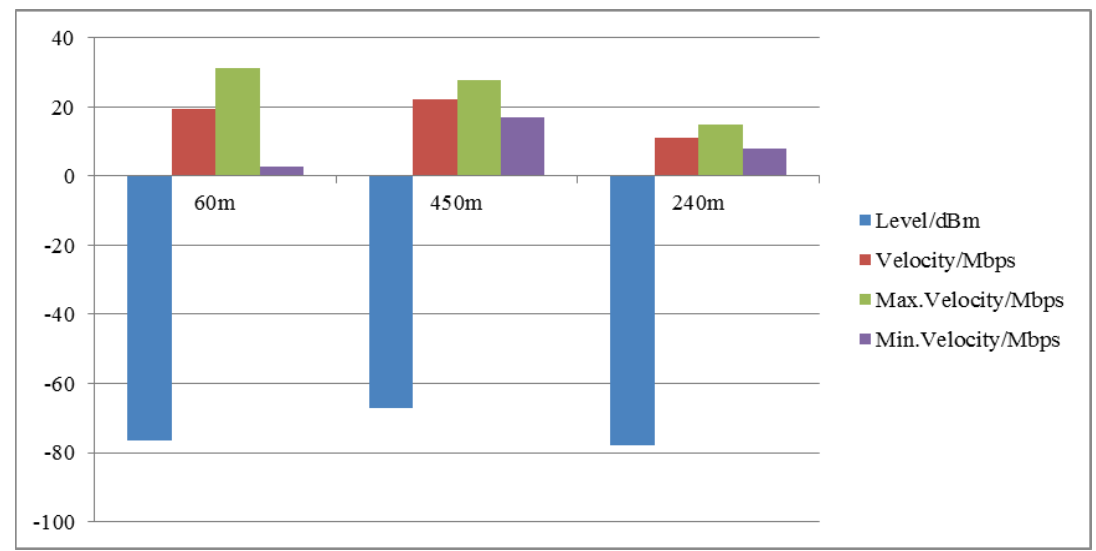

Figure 4. Wi-Fi test results 
PAPER

EMbedded Spectrum SEnsor Network Architecture And Transmission Medium TeSt Based ON TCP/IP

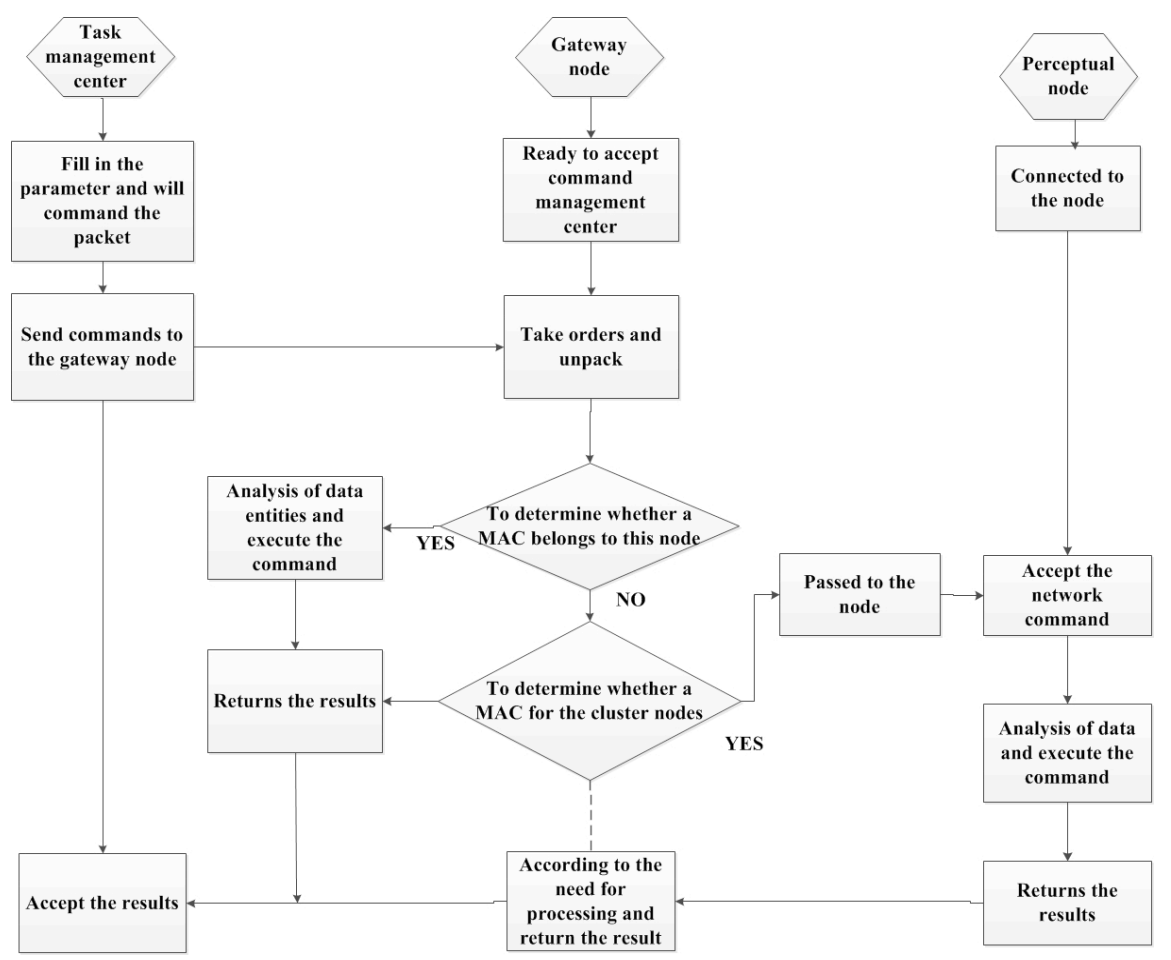

Figure 5. Flowchart of the implementation of the application layer protocol

\section{CONCLUSION}

Existing equipment cannot satisfy the increasing requirements of monitoring tasks. A feasible method to solve this problem is to use an electromagnetic spectrum monitoring equipment to set up a WSN.

This study analyzes the characteristics of a spectrummonitoring WSN by aiming at the actual demand for network operation. The data transmission speed and steady operation of network architecture are designed. The TCP/IP application layer is designed based on the analysis of TCP/IP.

This study designs the lower layer using TCP/IP and the upper layer using transport protocol according to the characteristics of spectrum-monitoring data in WSN. The lower layer cannot assure the effective supervision and retransmission mechanisms. By contrast, the design efficiency of the application layer protocol is high and can be extended in the future to yield sufficient space. The application layer protocol is implemented and tested in WSN. The test results validate its operation stability.

The proposed method uses an efficient transport protocol to achieve high-performance data transmission and to improve other aspects, including detection, scanning, positioning, and other business data transmission reliability and security.

\section{REFERENCES}

[1] Feiz, Atiyeh, et al. "Effect of different bleaching regimens on the flexural strength of hybrid composite resin", Journal of Conservative Dentistry, 2016, vol. 19, no. 2, pp. 157. http://dx.doi.org/10. 4103/0972-0707.178697

[2] Scherließ, Regina. "Inhalativa-Arzneiformen zur lokalen und systemischen Gabe Inhaled dosage forms-formulations for local and systemic delivery." PHARMAKON, 2016, vol. 4, no. 2, pp. 117-124.

[3] Olivares-Mendez, Miguel Angel, et al. "Vision-Based Steering Control, Speed Assistance and Localization for Inner-
CityVehicles", Sensors, 2016, vol. 16, no. 3, pp. 362. http://dx.doi.org/10.3390/s16030362

[4] Hara, Kosuke, and Hideo Saito. "Vehicle localization based on the detection of line segments from multi-camera images", Journal of Robotics and Mechatronics, 2015, vol. 27, no. 6, pp. 617-626. http://dx.doi.org/10.20965/jrm.2015.p0617

[5] Barman, Jahnabi, Reema Nath, and Lahari Saikia. "Drug resistance in Enterococcus species in a tertiary level hospital in Assam, India", The Indian Journal of Medical Research, 2016, vol. 143, no. 1, pp. 107. http://dx.doi.org/10.4103/0971-5916.178619

[6] McDowell, A., and M. Pai. "Treatment as diagnosis and diagnosis as treatment: empirical management of presumptive tuberculosis in India", The International Journal of Tuberculosis and Lung Disease, 2016, vol. 20, no. 4, pp. 536-543. http://dx.doi.org/10. 5588/ijtld.15.0562

[7] Li T, Meng Q, Liang J, et al. "Identification of five novel RB1 gene mutations in Chinese patients with retinoblastoma", Int $J$ Clin Exp Pathol, 2016, vol. 9, no.2, pp. 2120-2126.

[8] Gancheva D. "Intrahepatic cholestasis-clinical spectrum and therapeutic approach", Meditsinski Pregled/Medical Review, 2016, vol. 52, no. 1, pp. 10-23.

[9] Randis T M, Polin R A. "Neonatal medicine: past, present, and future", Current Opinion in Pediatrics, 2016, vol. 28, no. 2, pp. 133-134. http://dx.doi.org/10.1097/MOP.0000000000000342

[10] Yogi M, Surender K, Jadi L, et al. "Advantage of Computed Tomography (CT) Virtual Bronchoscopy in the Evaluation of Children with Suspected Foreign Body Airway", International Journal of Otolaryngology and Head \& Neck Surgery, 2016, vol. 5, no. 02, pp. 59. http://dx.doi.org/10.4236/ijohns.2016.52010

\section{AUTHORS}

Ya Zhang (corresponding author) is with the School of electronic and electrical engineering, Changzhou College of Information Technology, Changzhou, China (email: hnzy513@163.com).

Fang Han is with the College of mechanical and electrical engineering, Huainan Normal University, Huainan, China (e-mail: hanfang0554@126.com).

Submitted, 09 March 2016. Published as resubmitted by the authors on 09 April 2016. 